PROCEEDINGS OF THE

AMERICAN MATHEMATICAL SOCIETY

Volume 133, Number 11, Pages 3179-3189

S 0002-9939(05)07912-8

Article electronically published on May 9, 2005

\title{
AN EXTENSION OF WARNAAR'S MATRIX INVERSION
}

\author{
X. R. MA
}

(Communicated by John R. Stembridge)

\begin{abstract}
We present a necessary and sufficient condition for two matrices given by two bivariate functions to be inverse to each other with certainty in the cases of Krattenthaler formula and Warnaar's elliptic matrix inversion. Immediate consequences of our result are some known functions and a constructive approach to derive new matrix inversions from known ones.
\end{abstract}

\section{INTRODUCTION}

Throughout this paper, all operations are carried out on $\kappa$, a field of characteristic zero, and $Z$ denotes the set of nonnegative integers. Recall that $F=\left(f_{n, k}\right)_{n, k \in Z}$ is an infinite-dimensional lower-triangular matrix over $\kappa$ provided that each entry $f_{n, k}=0$ unless $n \geq k$. The matrix $G=\left(g_{n, k}\right)_{n, k \in Z}$ is the inverse matrix of $F$ if

$$
\sum_{n \geq i \geq k} f_{n, i} g_{i, k}=\delta_{n, k} \text { for all } n, k \in Z,
$$

where $\delta$ denotes the usual Kronecker delta. Two such matrices are often called an inversion or a reciprocal relation in the context of combinatorics. In what follows, we call a pair of matrices $F$ and $G$ with the reciprocal relation a matrix inversion. Matrix inversions play very important roles in many fields of combinatorics and special functions. For instance, we can use them to derive a number of basic hypergeometric summations and transformations. The reader may consult [1, 6, 13 , for more details. A well-known result concerning matrix inversions is the following formula discovered by Krattenthaler in [9], which is built on his earlier work of the operator method for solving Lagrange inversion problems [8] .

Theorem 1.1. Let $\left\{a_{i}\right\}_{i \in Z}$ and $\left\{b_{i}\right\}_{i \in Z}$ be arbitrary sequences over $\kappa$ such that $b_{i} \neq b_{j}$ for $i \neq j$. Assume that $F=\left(f_{n, k}\right)_{n, k \in Z}$ and $G=\left(g_{n, k}\right)_{n, k \in Z}$ are inverse to

Received by the editors May 4, 2004 and, in revised form, May 31, 2004, June 16, 2004 and June 22, 2004.

2000 Mathematics Subject Classification. Primary 05A10, 05A19, 33D15; Secondary 05A15, 33C20, 33D99.

Key words and phrases. Matrix inversion, elliptic hypergeometric series, Lagrange operator, kernel, antisymmetric, addition formula, polynomial transformation.

(C)2005 American Mathematical Society 
each other, where

$$
f_{n, k}=\frac{\prod_{j=k}^{n-1}\left(a_{j}-b_{k}\right)}{\prod_{j=k+1}^{n}\left(b_{j}-b_{k}\right)}
$$

Then

$$
g_{n, k}=\frac{a_{k}-b_{k}}{a_{n}-b_{n}} \frac{\prod_{j=k+1}^{n}\left(a_{j}-b_{n}\right)}{\prod_{j=k}^{n-1}\left(b_{j}-b_{n}\right)} .
$$

As many facts show, Krattenthaler's formula subsumes many classical matrix inversions in combinatorial analysis, such as Gould-Hsu inversion [7], Carlitz inversion (i.e., the $q$-analogue of Gould-Hsu inversion) [3], and Bressoud's formula [2].

Recently, as a new class of generalized hypergeometric series originally due to Frenkel and Turaev [5], the elliptic hypergeometric series has received much attention [5, 14, 17, 18]. In [18, Warnaar set up an elliptic matrix inversion by using the addition formula of the theta function [19] and by induction, which proved to be very useful in deriving an extensive list of summation and transformation formulas for elliptic hypergeometric series.

Theorem 1.2 (Warnaar's matrix inversion). Define the theta function $\theta(x)=$ $\prod_{j=0}^{\infty}\left(1-x p^{j}\right)\left(1-p^{j+1} / x\right),|p|<1$. Suppose that $F=\left(f_{n, k}\right)_{n, k \in Z}$ and $G=$ $\left(g_{n, k}\right)_{n, k \in Z}$ are inverse to each other. If

$$
\begin{aligned}
f_{n, k} & =\frac{\prod_{i=k}^{n-1} \theta\left(x_{i} b_{k}\right) \theta\left(x_{i} / b_{k}\right)}{\prod_{i=k+1}^{n} \theta\left(b_{i} b_{k}\right) \theta\left(b_{i} / b_{k}\right)}, \quad \text { then } \\
g_{n, k} & =\frac{b_{k} \theta\left(x_{k} b_{k}\right) \theta\left(x_{k} / b_{k}\right)}{b_{n} \theta\left(x_{n} b_{n}\right) \theta\left(x_{n} / b_{n}\right)} \frac{\prod_{i=k+1}^{n} \theta\left(x_{i} b_{n}\right) \theta\left(x_{i} / b_{n}\right)}{\prod_{i=k}^{n-1} \theta\left(b_{i} b_{n}\right) \theta\left(b_{i} / b_{n}\right)} .
\end{aligned}
$$

As was pointed out by Warnaar in [18], his matrix inversion is the elliptic analogue of Krattenthaler's formula. Also, it is worth mentioning that shortly after [18] appeared, Rosengren and Schlosser [14] reproved Warnaar's matrix inversion by using Krattenthaler's operator method.

In this paper, we will show that Warnaar's matrix inversion admits a further extension, namely, there exists a new unified matrix inversion which contains Warnaar's matrix inversion (thus, Krattenthaler's formula) as a special case. More precisely, once the addition formula of the theta function is extended to the kernel of the Lagrange operator (see Definition 2.3 below), can we establish the following new matrix inversion containing $(1.1) /(1.2)$ and $(1.3) /(1.4)$ as special cases, which will be proved in section 3 .

Theorem 1.3. Let $f(x, y)$ and $g(x, y)$ be two arbitrary functions over $\kappa$ in variables $x, y$. Suppose further $g(x, y)$ is antisymmetric, i.e., $g(x, y)=-g(y, x)$. Let $F=$ 
$\left(f_{n, k}\right)_{n, k \in Z}$ and $G=\left(g_{n, k}\right)_{n, k \in Z}$ be two matrices with entries given by

$$
\begin{aligned}
f_{n, k} & =\frac{\prod_{i=k}^{n-1} f\left(x_{i}, b_{k}\right)}{\prod_{i=k+1}^{n} g\left(b_{i}, b_{k}\right)} \quad \text { and } \\
g_{n, k} & =\frac{f\left(x_{k}, b_{k}\right)}{f\left(x_{n}, b_{n}\right)} \frac{\prod_{i=k+1}^{n} f\left(x_{i}, b_{n}\right)}{\prod_{i=k}^{n-1} g\left(b_{i}, b_{n}\right)}, \text { respectively. }
\end{aligned}
$$

Then $F=\left(f_{n, k}\right)_{n, k \in Z}$ and $G=\left(g_{n, k}\right)_{n, k \in Z}$ is a matrix inversion if and only if for all $a, b, c, x \in \kappa$,

$$
g(a, b) f(x, c)-g(a, c) f(x, b)+g(b, c) f(x, a)=0 .
$$

Here and in what follows, it is always assumed that $\left\{b_{i}\right\}_{i \in Z}$ is a sequence such that none of the terms $g\left(b_{j}, b_{i}\right)$ or $f\left(x_{j}, b_{i}\right)$ in the denominator of the right-hand side vanishes, which is no longer listed as a condition. Besides, any product of the form $\prod_{i=k}^{n} \bullet$ while $k>n$ is defined to be 1 . As we will see later, (1.7) can be regarded as a generalization of the addition formula of the theta function.

\section{Definitions AND PRELIMINARY RESUlts}

Let us begin with two Lagrange operators on which the proof of Theorem 1.3 relies entirely. To do this, we use $\kappa^{n}$ to denote the $n$-dimensional vector space over $\kappa$, and $\kappa\left(x_{1}, x_{2}, \cdots, x_{n}\right)$ the set of all functions over $\kappa$ in variables $x_{1}, x_{2}, \cdots, x_{n}$.

Definition 2.1 (Lagrange operator). Let $\kappa_{0}(x, y)$ be the set of antisymmetric bivariate functions $f(x, y)$. Then the map $\mathcal{L}_{n}: \kappa^{n} \times \kappa_{0}(x, y) \longrightarrow \kappa\left(x_{1}, x_{2}, \cdots, x_{n-2}\right)$, such that

$$
\mathcal{L}_{n}\left(b_{1}, b_{2}, \cdots, b_{n}, f\right)=\sum_{i=1}^{n}(-1)^{n-i} \prod_{1 \leq i_{1}<i_{2} \leq n ; i_{1}, i_{2} \neq i} f\left(b_{i_{1}}, b_{i_{2}}\right) \prod_{j=1}^{n-2} f\left(x_{j}, b_{i}\right),
$$

is said to be the Lagrange operator of degree $n$.

Definition 2.2 (Generalized Lagrange operator). Let $\kappa_{0}(x, y)$ be defined as above, $g(x, y) \in \kappa_{0}(x, y)$. Then the map $\mathcal{L}_{n}^{(g)}: \kappa^{n} \times \kappa(x, y) \longrightarrow \kappa\left(x_{1}, x_{2}, \cdots, x_{n-2}\right)$, such that

$$
\mathcal{L}_{n}^{(g)}\left(b_{1}, b_{2}, \cdots, b_{n}, f\right)=\sum_{i=1}^{n}(-1)^{n-i} \prod_{1 \leq i_{1}<i_{2} \leq n ; i_{1}, i_{2} \neq i} g\left(b_{i_{1}}, b_{i_{2}}\right) \prod_{j=1}^{n-2} f\left(x_{j}, b_{i}\right),
$$

is said to be the generalized Lagrange operator of degree $n$ with respect to $g(x, y)$.

Evidently, $\mathcal{L}_{n} \neq \mathcal{L}_{n}^{(g)}$, although $\mathcal{L}_{n}\left(b_{1}, b_{2}, \cdots, b_{n}, f\right)=\mathcal{L}_{n}^{(f)}\left(b_{1}, b_{2}, \cdots, b_{n}, f\right)$. We will write $\mathcal{L}_{n}\left(b_{1}, b_{2}, \cdots, b_{n}\right)(f)$ for $\mathcal{L}_{n}\left(b_{1}, b_{2}, \cdots, b_{n}, f\right), \mathcal{L}_{n}^{(g)}\left(b_{1}, b_{2}, \cdots, b_{n}\right)(f)$ for $\mathcal{L}_{n}^{(g)}\left(b_{1}, b_{2}, \cdots, b_{n}, f\right)$ for short. In particular, $\mathcal{L}_{1}\left(b_{1}\right)(f)=\mathcal{L}_{1}^{(g)}\left(b_{1}\right)(f)=1$, $\mathcal{L}_{2}\left(b_{1}, b_{2}\right)(f)=\mathcal{L}_{2}^{(g)}\left(b_{1}, b_{2}\right)(f)=0$, all of which follow from the previous assumption and definitions.

Example 2.1. For all $(a, b, c, d) \in \kappa^{4}, g(x, y) \in \kappa_{0}(x, y)$, the action of the Lagrange operator $\mathcal{L}_{4}^{(g)}$ of degree 4 on $f(x, y)$ is by Definition 2.2 the following:

$$
\begin{aligned}
\mathcal{L}_{4}^{(g)} & (a, b, c, d)(f)=g(a, b) g(a, c) g(b, c) f\left(x_{1}, d\right) f\left(x_{2}, d\right) \\
& -g(a, b) g(a, d) g(b, d) f\left(x_{1}, c\right) f\left(x_{2}, c\right)+g(a, c) g(a, d) g(c, d) f\left(x_{1}, b\right) f\left(x_{2}, b\right) \\
& -g(b, c) g(c, d) g(b, d) f\left(x_{1}, a\right) f\left(x_{2}, a\right) .
\end{aligned}
$$


Next, we are concerned with a function $f(x, y)$ such that $\mathcal{L}_{n}\left(b_{1}, b_{2}, \cdots, b_{n}\right)(f)=$ 0 or $\mathcal{L}_{n}^{(g)}\left(b_{1}, b_{2}, \cdots, b_{n}\right)(f)=0$. For this, we need the following definition:

Definition 2.3. Let $\mathcal{L}_{n}$ and $\mathcal{L}_{n}^{(g)}$ be defined as above. Then the kernel of $\mathcal{L}_{n}$ (resp. $\left.\mathcal{L}_{n}^{(g)}\right)$ is defined to be the set of all bivariate functions $f(x, y)$ such that for any $\left(b_{1}, b_{2}, \cdots, b_{n}\right) \in \kappa^{n}, \mathcal{L}_{n}\left(b_{1}, b_{2}, \cdots, b_{n}\right)(f)=0\left(\right.$ resp. $\left.\mathcal{L}_{n}^{(g)}\left(b_{1}, b_{2}, \cdots, b_{n}\right)(f)=0\right)$.

For convenience, the kernels of $\mathcal{L}_{n}$ and $\mathcal{L}_{n}^{(g)}$ are denoted respectively by $\operatorname{Ker} \mathcal{L}_{n}$ and $\operatorname{Ker} \mathcal{L}_{n}^{(g)}$.

Lemma 2.1. With the definitions and notation above, the following are valid for all integer $n \geq 4$ and $b_{1}, b_{2}, \cdots, b_{n} \in \kappa$ :

$$
\begin{array}{r}
\left.\mathcal{L}_{n}\left(b_{1}, b_{2}, \cdots, b_{n}\right)(f)\right|_{x_{n-2}=b_{n}}=\prod_{i=1}^{n-1} f\left(b_{i}, b_{n}\right) \times \mathcal{L}_{n-1}\left(b_{1}, b_{2}, \cdots, b_{n-1}\right)(f) ; \\
\mathcal{L}_{n}\left(b_{1}, b_{2}, \cdots, b_{n}\right)(f)=f\left(x_{n-2}, b_{n}\right) \prod_{i=2}^{n-1} f\left(b_{1}, b_{i}\right) \times \mathcal{L}_{n-1}\left(b_{2}, b_{3}, \cdots, b_{n}\right)(f) \\
\text { provided that } \quad \mathcal{L}_{n-1}\left(b_{1}, b_{2}, \cdots, b_{n-1}\right)(f)=0 ; \\
\mathcal{L}_{n}^{(g)}\left(b_{1}, b_{2}, \cdots, b_{n}\right)(f)=f\left(x_{n-2}, b_{n}\right) \prod_{i=2}^{n-1} g\left(b_{1}, b_{i}\right) \times \mathcal{L}_{n-1}^{(g)}\left(b_{2}, b_{3}, \cdots, b_{n}\right)(f) \\
\text { provided that } \mathcal{L}_{n-1}^{(g)}\left(b_{1}, b_{2}, \cdots, b_{n-1}\right)(f)=0, \text { and } f(x, y) \in \operatorname{KerL}_{3}^{(g)} .
\end{array}
$$

Here, $\left.\mathcal{L}_{n}\left(b_{1}, b_{2}, \cdots, b_{n}\right)(f)\right|_{x_{n-2}=b_{n}}$ stands for the value of $\mathcal{L}_{n}\left(b_{1}, b_{2}, \cdots, b_{n}\right)(f)$ with the restriction that $x_{n-2}=b_{n}$.

Proof. For simplicity, we only show (2.1) and (2.3); (2.2) can be proved by the same sort of argument as in (2.3), which is omitted here.

The proof of (2.1). By Definition 2.1 it is easily seen that

$$
\begin{aligned}
& \mathcal{L}_{n}\left(b_{1}, b_{2}, \cdots, b_{n-1}, b_{n}\right)(f) \\
& =\sum_{i=1}^{n}(-1)^{n-i} \prod_{1 \leq i_{1}<i_{2} \leq n ; i_{1}, i_{2} \neq i} f\left(b_{i_{1}}, b_{i_{2}}\right) f\left(x_{n-2}, b_{i}\right) \prod_{j=1}^{n-3} f\left(x_{j}, b_{i}\right) .
\end{aligned}
$$

Let $x_{n-2}=b_{n}$ in (2.4). In this case, $f\left(x_{n-2}, b_{n}\right)=0$. Thus, (2.4) reduces to

$$
\begin{aligned}
& \left.\mathcal{L}_{n}\left(b_{1}, b_{2}, \cdots, b_{n-1}, b_{n}\right)(f)\right|_{x_{n-2}=b_{n}} \\
= & \sum_{i=1}^{n-1}(-1)^{n-i} \prod_{1 \leq i_{1}<i_{2} \leq n ; i_{1}, i_{2} \neq i} f\left(b_{i_{1}}, b_{i_{2}}\right) f\left(b_{n}, b_{i}\right) \prod_{j=1}^{n-3} f\left(x_{j}, b_{i}\right) .
\end{aligned}
$$

Observe that

$$
\begin{aligned}
& (-1)^{n-i} \prod_{1 \leq i_{1}<i_{2} \leq n ; i_{1}, i_{2} \neq i} f\left(b_{i_{1}}, b_{i_{2}}\right) f\left(b_{n}, b_{i}\right) \\
= & (-1)^{n-1-i} \prod_{1 \leq i_{1}<i_{2} \leq n-1 ; i_{1}, i_{2} \neq i} f\left(b_{i_{1}}, b_{i_{2}}\right) \prod_{1 \leq j \leq n-1} f\left(b_{j}, b_{n}\right) .
\end{aligned}
$$


Setting this result into (2.5) and making some simplification, we get

$$
\begin{aligned}
& \left.\mathcal{L}_{n}\left(b_{1}, b_{2}, \cdots, b_{n-1}, b_{n}\right)(f)\right|_{x_{n-2}=b_{n}} \\
= & \prod_{1 \leq i \leq n-1} f\left(b_{i}, b_{n}\right) \sum_{i=1}^{n-1}(-1)^{n-1-i} \prod_{1 \leq i_{1}<i_{2} \leq n-1 ; i_{1}, i_{2} \neq i} f\left(b_{i_{1}}, b_{i_{2}}\right) \prod_{j=1}^{n-3} f\left(x_{j}, b_{i}\right) \\
= & \prod_{1 \leq i \leq n-1} f\left(b_{i}, b_{n}\right) \mathcal{L}_{n-1}\left(b_{1}, b_{2}, \cdots, b_{n-1}\right)(f),
\end{aligned}
$$

which is the desired right-hand side of (2.1).

The proof of (2.3). Now, by Definition 2.2.

$$
\begin{aligned}
\mathcal{L}_{n}^{(g)} & \left(b_{1}, b_{2}, \cdots, b_{n-1}, b_{n}\right)(f) \\
= & \sum_{i=1}^{n}(-1)^{n-i} \prod_{1 \leq i_{1}<i_{2} \leq n ; i_{1}, i_{2} \neq i} g\left(b_{i_{1}}, b_{i_{2}}\right) \prod_{j=1}^{n-2} f\left(x_{j}, b_{i}\right) \\
= & \prod_{j=1}^{n-2} f\left(x_{j}, b_{n}\right) \prod_{1 \leq i_{1}<i_{2} \leq n-1} g\left(b_{i_{1}}, b_{i_{2}}\right) \\
& +\sum_{i=2}^{n-1}(-1)^{n-i} \prod_{j=1}^{n-3} f\left(x_{j}, b_{i}\right) \prod_{1 \leq i_{1}<i_{2} \leq n ; i_{1}, i_{2} \neq i} g\left(b_{i_{1}}, b_{i_{2}}\right) f\left(x_{n-2}, b_{i}\right) \\
& +\left\{(-1)^{n-1} \prod_{j=1}^{n-3} f\left(x_{j}, b_{1}\right) \prod_{2 \leq i_{1}<i_{2} \leq n-1} g\left(b_{i_{1}}, b_{i_{2}}\right)\right\} f\left(x_{n-2}, b_{1}\right) \prod_{2 \leq i \leq n-1} g\left(b_{i}, b_{n}\right) .
\end{aligned}
$$

Furthermore, a slight transformation on the known condition,

$$
\mathcal{L}_{n-1}^{(g)}\left(b_{1}, b_{2}, \cdots, b_{n-1}\right)(f)=0
$$

leads to

$$
\begin{aligned}
(-1)^{n-1} & \prod_{j=1}^{n-3} f\left(x_{j}, b_{1}\right) \prod_{2 \leq i_{1}<i_{2} \leq n-1} g\left(b_{i_{1}}, b_{i_{2}}\right) \\
= & \sum_{i=2}^{n-1}(-1)^{n-1-i} \prod_{1 \leq i_{1}<i_{2} \leq n-1 ; i_{1}, i_{2} \neq i} g\left(b_{i_{1}}, b_{i_{2}}\right) \prod_{j=1}^{n-3} f\left(x_{j}, b_{i}\right) .
\end{aligned}
$$


Inserting this in the above identity and making some simplification yields that

$$
\begin{aligned}
& \mathcal{L}_{n}^{(g)}\left(b_{1}, b_{2}, \cdots, b_{n-1}, b_{n}\right)(f)=\prod_{1 \leq i_{1}<i_{2} \leq n-1} g\left(b_{i_{1}}, b_{i_{2}}\right) \prod_{j=1}^{n-2} f\left(x_{j}, b_{n}\right) \\
+ & \sum_{i=2}^{n-1}(-1)^{n-i} \prod_{j=1}^{n-3} f\left(x_{j}, b_{i}\right)\left\{f\left(x_{n-2}, b_{i}\right) \prod_{1 \leq i_{1}<i_{2} \leq n ; i_{1}, i_{2} \neq i} g\left(b_{i_{1}}, b_{i_{2}}\right)\right. \\
& \left.-f\left(x_{n-2}, b_{1}\right) \prod_{2 \leq j \leq n-1} g\left(b_{j}, b_{n}\right) \prod_{1 \leq i_{1}<i_{2} \leq n-1 ; i_{1}, i_{2} \neq i} g\left(b_{i_{1}}, b_{i_{2}}\right)\right\} \\
= & \prod_{1 \leq i_{1}<i_{2} \leq n-1} g\left(b_{i_{1}}, b_{i_{2}}\right) \prod_{j=1}^{n-2} f\left(x_{j}, b_{n}\right)+\sum_{i=2}^{n-1}(-1)^{n-i} \prod_{j=1}^{n-3} f\left(x_{j}, b_{i}\right) \prod_{j=2, j \neq i}^{n-1} g\left(b_{j}, b_{n}\right) \\
& \times \prod_{1 \leq i_{1}<i_{2} \leq n-1 ; i_{1}, i_{2} \neq i} g\left(b_{i_{1}}, b_{i_{2}}\right)\left\{g\left(b_{1}, b_{n}\right) f\left(x_{n-2}, b_{i}\right)-g\left(b_{i}, b_{n}\right) f\left(x_{n-2}, b_{1}\right)\right\} .
\end{aligned}
$$

Note that $f(x, y) \in \operatorname{Ker} \mathcal{L}_{3}^{(g)}$. Thus, for $\left(b_{1}, b_{i}, b_{n}\right) \in \kappa^{3}$, it also holds that

$$
g\left(b_{1}, b_{n}\right) f\left(x_{n-2}, b_{i}\right)-g\left(b_{i}, b_{n}\right) f\left(x_{n-2}, b_{1}\right)=g\left(b_{1}, b_{i}\right) f\left(x_{n-2}, b_{n}\right) .
$$

Moreover, it is easily seen that

$$
\begin{aligned}
& g\left(b_{1}, b_{i}\right) \prod_{j=2, j \neq i}^{n-1} g\left(b_{j}, b_{n}\right) \prod_{1 \leq i_{1}<i_{2} \leq n-1 ; i_{1}, i_{2} \neq i} g\left(b_{i_{1}}, b_{i_{2}}\right) \\
& =\prod_{j=2}^{n-1} g\left(b_{1}, b_{j}\right) \prod_{2 \leq i_{1}<i_{2} \leq n ; i_{1}, i_{2} \neq i} g\left(b_{i_{1}}, b_{i_{2}}\right) .
\end{aligned}
$$

Thus, to simplify the preceding identity by inserting these, we get

$$
\begin{array}{r}
\mathcal{L}_{n}^{(g)}\left(b_{1}, b_{2}, \cdots, b_{n-1}, b_{n}\right)(f)=\prod_{1 \leq i_{1}<i_{2} \leq n-1} g\left(b_{i_{1}}, b_{i_{2}}\right) \prod_{j=1}^{n-2} f\left(x_{j}, b_{n}\right) \\
+f\left(x_{n-2}, b_{n}\right) \prod_{j=2}^{n-1} g\left(b_{1}, b_{j}\right) \sum_{i=2}^{n-1}(-1)^{n-i} \prod_{j=1}^{n-3} f\left(x_{j}, b_{i}\right) \prod_{2 \leq i_{1}<i_{2} \leq n ; i_{1}, i_{2} \neq i} g\left(b_{i_{1}}, b_{i_{2}}\right) .
\end{array}
$$

Note that

$$
\prod_{1 \leq i_{1}<i_{2} \leq n-1} g\left(b_{i_{1}}, b_{i_{2}}\right)=\prod_{j=2}^{n-1} g\left(b_{1}, b_{j}\right) \prod_{2 \leq i_{1}<i_{2} \leq n ; i_{1}, i_{2} \neq n} g\left(b_{i_{1}}, b_{i_{2}}\right) .
$$

When inserted in (2.6) and rearranged, it follows that

$$
\begin{aligned}
& \mathcal{L}_{n}^{(g)}\left(b_{1}, b_{2}, \cdots, b_{n-1}, b_{n}\right)(f) \\
= & f\left(x_{n-2}, b_{n}\right) \prod_{j=2}^{n-1} g\left(b_{1}, b_{j}\right) \sum_{i=2}^{n}(-1)^{n-i} \prod_{2 \leq i_{1}<i_{2} \leq n ; i_{1}, i_{2} \neq i} g\left(b_{i_{1}}, b_{i_{2}}\right) \prod_{j=1}^{n-3} f\left(x_{j}, b_{i}\right) \\
= & f\left(x_{n-2}, b_{n}\right) \prod_{j=2}^{n-1} g\left(b_{1}, b_{j}\right) \mathcal{L}_{n-1}^{(g)}\left(b_{2}, b_{3}, \cdots, b_{n}\right)(f), \quad \text { as desired. }
\end{aligned}
$$

Lemma 2.1 provides a full characterization of the kernels of Lagrange operators. 
Theorem 2.1. Let $\mathcal{L}_{n}$ and $\mathcal{L}_{n}^{(g)}, \operatorname{Ker} \mathcal{L}_{n}$ and $\operatorname{Ker} \mathcal{L}_{n}^{(g)}$ be defined as above. Then for any integer $n \geq 4$, the following hold:

$$
\text { (i) } \operatorname{KerL}_{n-1}=\operatorname{KerL}_{n} ; \quad \text { (ii) } \operatorname{KerL}_{n-1}^{(g)} \subseteq \operatorname{KerL}_{n}^{(g)} .
$$

Proof. (i) Clearly, the identity (2.2) of Lemma 2.1] implies that $\operatorname{Ker} \mathcal{L}_{n-1} \subseteq \operatorname{Ker} \mathcal{L}_{n}$. On the other hand, if $f(x, y) \in \operatorname{Ker} \mathcal{L}_{n}$, then by Definition 2.3, we see that for all $\left(b_{1}, b_{2}, \cdots, b_{n}\right) \in \kappa^{n}$,

$$
\mathcal{L}_{n}\left(b_{1}, b_{2}, \cdots, b_{n}\right)(f)=0 .
$$

Hence, setting $x_{n-2}=b_{n}$ in (2.7) and by the identity (2.1), we obtain directly that $\mathcal{L}_{n-1}\left(b_{1}, b_{2}, \cdots, b_{n-1}\right)(f)=0$, i.e., $f(x, y) \in \operatorname{Ker} \mathcal{L}_{n-1}$, which means that $\operatorname{Ker} \mathcal{L}_{n} \subseteq \operatorname{Ker} \mathcal{L}_{n-1}$. Summing up, $\operatorname{Ker} \mathcal{L}_{n-1}=\operatorname{Ker} \mathcal{L}_{n}$.

(ii) Given $f(x, y) \in \operatorname{Ker} \mathcal{L}_{3}^{(g)}$, it is easy to check that $f(x, y) \in \operatorname{Ker} \mathcal{L}_{4}^{(g)}$. Therefore, starting from this and using the identity (2.3), the desired result follows easily by induction.

Corollary 2.1. Let $\mathcal{L}_{n}$ and $\mathcal{L}_{n}^{(g)}, \operatorname{Ker} \mathcal{L}_{n}$ and $\operatorname{Ker} \mathcal{L}_{n}^{(g)}$ be defined as above. Then for any integer $n \geq 3$,

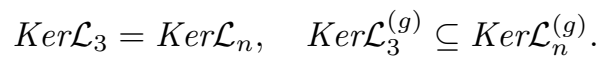

\section{The Proof of Theorem 1.3}

Now, we are fully prepared to give the proof of the main theorem.

The proof of Theorem 1.3. First, assume that $f(x, y) \in \operatorname{Ker} \mathcal{L}_{3}^{(g)}$. Now, to show $F=\left(f_{n, k}\right)_{n, k \in Z}$ and $G=\left(g_{n, k}\right)_{n, k \in Z}$ with entries given by (1.5) and (1.6), respectively, are inverse to each other, it suffices to show

$$
\sum_{i=k}^{n} f_{n, i} g_{i, k}=\delta_{n, k}
$$

Obviously, by our assumption, (3.1) is valid for $n-k \leq 1$. Thus, it suffices to show $\sum_{i=k}^{n} f_{n, i} g_{i, k}=0$ for $n-k \geq 2$. To do this, we compute

$$
\sum_{i=k}^{n} f_{n, i} g_{i, k}=f\left(x_{k}, b_{k}\right) \times \sum_{i=k}^{n} \frac{\prod_{j=k+1}^{n-1} f\left(x_{j}, b_{i}\right)}{\prod_{j=k, j \neq i}^{n} g\left(b_{j}, b_{i}\right)} .
$$

Therefore, we need only to show

$$
\sum_{i=k}^{n} \frac{\prod_{j=k+1}^{n-1} f\left(x_{j}, b_{i}\right)}{\prod_{j=k, j \neq i}^{n} g\left(b_{j}, b_{i}\right)}=0 .
$$

For this, to multiply both sides of (3.2) by $\prod_{k \leq i_{1}<i_{2} \leq n} g\left(b_{i_{1}}, b_{i_{2}}\right)$, and then to simplify by $g\left(b_{i}, b_{j}\right)=-g\left(b_{j}, b_{i}\right)$, we get another equivalent form of (3.2),

$$
\sum_{i=k}^{n}(-1)^{n-k+1-i} \prod_{k \leq i_{1}<i_{2} \leq n, i_{1}, i_{2} \neq i} g\left(b_{i_{1}}, b_{i_{2}}\right) \prod_{j=k+1}^{n-1} f\left(x_{j}, b_{i}\right)=0,
$$

which can be restated in terms of generalized Lagrange operator $\mathcal{L}_{n}^{(g)}$ as follows:

$$
\mathcal{L}_{n-k+1}^{(g)}\left(b_{k}, b_{k+1}, \cdots, b_{n}\right)(f)=\delta_{n, k} \quad \text { for } \quad n-k \geq 2,
$$


i.e., $\quad f(x, y) \in \operatorname{Ker}_{\mathcal{L}_{-k+1}^{(g)}}, n-k+1 \geq 3$, which is guaranteed by Corollary 2.1 and the known condition $f(x, y) \in \operatorname{Ker}_{3}^{(g)}$. Conversely, assume that $F=\left(f_{n, k}\right)_{n, k \in Z}$ and $G=\left(g_{n, k}\right)_{n, k \in Z}$ with entries given by (1.5) and (1.6), respectively, are inverse to each other. Then it holds that

$$
\mathcal{L}_{n-k+1}^{(g)}\left(b_{k}, b_{k+1}, \cdots, b_{n}\right)(f)=\delta_{n, k} \quad \text { for } \quad n-k \geq 2,
$$

that is, $\quad f(x, y) \in \operatorname{Ker}_{n}^{(g)} \mathcal{L}_{n+1}, n-k+1 \geq 3$, which covers the desired result. Summing up, we have finished the complete proof of theorem.

Remark 3.1. The referee pointed out that Theorem 1.3 can also be proved by adapting Warnaar's inductive proof. We also tried Krattenthaler's operator method, but succeeded only in the special case that $f(x, y)=g(x, y)$. But here, we prefer the above operator proof because we believe it is helpful and suggestive for finding any multidimensional form of Theorem 1.3 .

\section{Some explicit functions of $\operatorname{Ker} \mathcal{L}_{3}$ And $\operatorname{Ker} \mathcal{L}_{3}^{(g)}$}

Theorem 1.3 clearly states that the matrix inversion $(1.5) /(1.6)$ depends on each function of $\operatorname{Ker} \mathcal{L}_{3}$ or $\operatorname{Ker} \mathcal{L}_{3}^{(g)}$. Thus, it is a very worthwhile research problem to find a complete characterization of the functions $f(x, y)$ in these two kernels. While we are yet not able to do it in full generality, we give three families of such functions in the next theorem.

Theorem 4.1. Let $\operatorname{KerL}_{3}$ and $\operatorname{KerL}_{3}^{(g)}$ be defined as before. Then the following hold:

(i) If $g(x, y)=(x-y)(1-a x y)$, then $(1-x y)(x-a y) \in \operatorname{KerL}_{3}^{(g)}$.

(ii) Let $\theta(x)$ be defined as in Theorem 1.2, $f(x, y)=y \theta(x y) \theta(x / y)$. Then $f(x, y) \in \operatorname{KerL}_{3}$.

(iii) Let $n_{i}(i=1,2, \cdots, m)$ be nonnegative integers such that $n_{i}<n_{j}(i<j)$, and $a_{i} \in \kappa(i=1,2, \cdots, 2 m-3)$ arbitrary constants. Then

$$
\begin{array}{r}
f(x, y)=\sum_{i=1}^{2} \sum_{j=i+1}^{m} a_{1} a_{m(i-1)+j-2 i+1}\left(x^{n_{i}} y^{n_{j}}-x^{n_{j}} y^{n_{i}}\right) \\
+\sum_{i=3}^{m-1} \sum_{j=i+1}^{m}\left(a_{i-1} a_{m+j-3}-a_{j-1} a_{m+i-3}\right)\left(x^{n_{i}} y^{n_{j}}-x^{n_{j}} y^{n_{i}}\right) \in \text { KerL } \mathcal{L}_{3} .
\end{array}
$$

Proof. (i) This can be verified in a straightforward way and is omitted here.

(ii) For this case, it suffices to check that $f(x, y)=-f(y, x)$ and the special case $f(x, y)=g(x, y)$ of (1.7) holds for all $a, b, c, x \in \kappa$. Actually, from the property that $\theta(x)=-x \theta(1 / x)$, we find that

$$
f(x, y) / f(y, x)=\frac{y \theta(x / y)}{x \theta(y / x)}=-1, \text { i.e., } f(x, y)=-f(y, x)
$$

On the other hand, the addition formula of the theta function [19] states that for all $a, b, c \in \kappa$,

$$
\theta(a b, a / b, x c, x / c)-\theta(a c, a / c, x b, x / b)=\frac{x}{b} \theta(b c, b / c, x a, a / x),
$$


where $\theta\left(x_{1}, x_{2}, x_{3}, x_{4}\right)=\theta\left(x_{1}\right) \theta\left(x_{2}\right) \theta\left(x_{3}\right) \theta\left(x_{4}\right)$. Replacing $\theta$ in the addition formula (4.2) by $f(x, y)$, we obtain that

$$
\begin{aligned}
& \frac{1}{b} f(a, b) \frac{1}{c} f(x, c)-\frac{1}{c} f(a, c) \frac{1}{b} f(x, b)=\frac{x}{b} \frac{1}{c} f(b, c) \frac{1}{x} f(a, x), \text { i.e., } \\
& f(a, b) f(x, c)-f(a, c) f(x, b)+f(b, c) f(x, a)=0, \quad \text { as desired. }
\end{aligned}
$$

(iii) In this case, we might assume that $f(x, y)=\sum_{0 \leq r<s \leq+\infty} \lambda_{r, s}\left(x^{r} y^{s}-x^{s} y^{r}\right)$, where

$$
\lambda_{r, s}= \begin{cases}a_{1} a_{m(i-1)+j-2 i+1}, & r=n_{i}, s=n_{j}, i=1,2, i<j \leq m \\ a_{i-1} a_{m+j-3}-a_{j-1} a_{m+i-3}, & r=n_{i}, s=n_{j}, 3 \leq i<j \leq m \\ 0, & \text { otherwise. }\end{cases}
$$

Observe that to show $f(x, y) \in \operatorname{Ker} \mathcal{L}_{3}$ amounts to checking that the special case $f(x, y)=g(x, y)$ of (1.7) holds for all $a, b, c, x \in \kappa$, that is, for all nonnegative integers $p, q, r, s$, the coefficient of term $a^{p} b^{q} c^{s} x^{r}$ in the left-hand side of (1.7) is zero. In fact, we need only to check that for all $p, q, r, s \in\left\{n_{1}, n_{2}, \cdots, n_{m}\right\}$,

$$
\lambda_{p, q} \lambda_{r, s}-\lambda_{p, s} \lambda_{r, q}+\lambda_{q, s} \lambda_{r, p}=0
$$

which can be verified by direct calculation and is left to the reader.

Now we point out some relevant remarks on Theorem 4.1

Remark 4.1. As an application of the case (iii), if we take in (4.1) $m=2, n_{1}=$ $0, n_{2}=1, a_{1}=-1$, then $f(x, y)=x-y$. Note that $g(x, y)=f(x, y)$. Thus, (1.5)/(1.6) in Theorem [1.3 precisely yields Krattenthaler formula; Theorem 1.3 in the special case (i) corresponds to Krattenthaler's corollary (see 9], (1.5)(1)/ $(1.5)(2))$; Theorem 1.3 corresponding to $f(x, y)=g(x, y)=y \theta(x y) \theta(x / y)$ is exactly Warnaar's matrix inversion. Thus, it is reasonable to say that (1.7) is a generalization of the addition formula of the theta function. On the other hand, since the function $f(x, y)$ in (iii) cannot always be expressed as the form in (ii) in terms of the theta function $\theta$, Theorem 1.3 contains many matrix inversions not being covered by Warnaar's matrix inversion. In other words, Theorem 1.3 is indeed a nontrivial generalization of Warnaar's matrix inversion.

Further, if we still restrict ourselves to polynomials, then we can set up a constructive way to derive new matrix inversions from known ones. For this, we need the notation $\kappa[x, y]$ and $\Lambda_{3}[x, y]$ (resp. $\Lambda_{3}^{(g)}[x, y]$ ) to denote the ring of polynomials over $\kappa$ and the set of all polynomials contained in $\operatorname{KerL}_{3}$ (resp. $\operatorname{Ker} \mathcal{L}_{3}^{(g)}$ ), respectively. Obviously, $\Lambda_{3}^{(g)}[x, y]$ is a linear subspace of $\operatorname{Ker} \mathcal{L}_{3}^{(g)}$.

Definition 4.1. Suppose $\Phi$ is a map from $\kappa[x, y]$ to itself. If there exists a positive integer $p$ and $a_{i} \in \kappa, i=0,1, \cdots, p$, such that for all $f(x, y) \in \kappa[x, y]$

$$
\Phi(f)(x, y)=\sum_{0 \leq i, j \leq p} f\left(a_{i}, a_{j}\right) x^{i} y^{j}
$$

then $\Phi$ is said to be a polynomial transformation over $\kappa[x, y]$.

Theorem 4.2. Let $\Lambda_{3}^{(g)}[x, y] \subseteq \operatorname{Ker}_{3}^{(g)}$ and $\Phi$ be a polynomial transformation, defined as above. Then $\Phi$ is a linear transformation from $\Lambda_{3}^{(g)}[x, y]$ to $\Lambda_{3}^{(\Phi(g))}[x, y]$. 
Proof. Since $\Phi$ is a polynomial transformation, by Definition 4.1, there exists a positive integer $p$ and $a_{i} \in \kappa, i=0,1, \cdots, p$, such that

$$
\Phi(f)(x, y)=\sum_{0 \leq i, j \leq p} f\left(a_{i}, a_{j}\right) x^{i} y^{j} .
$$

Obviously, $\Phi$ is linear and $\Phi(g)(x, y)$ is an antisymmetric polynomial. Thus, $\mathcal{L}_{3}^{(\Phi(g))}$ is well defined. We need only to show that $\Phi(f)(x, y) \in \operatorname{Ker} \mathcal{L}_{3}^{(\Phi(g))}$, that is, for $a, b, c, x \in \kappa$,

$$
\Phi(g)(a, b) \Phi(f)(x, c)-\Phi(g)(a, c) \Phi(f)(x, b)+\Phi(g)(b, c) \Phi(f)(x, a)=0 .
$$

To do this, we compute the left-hand side of 4.3):

$$
\begin{aligned}
& \Phi(g)(a, b) \Phi(f)(x, c)-\Phi(g)(a, c) \Phi(f)(x, b)+\Phi(g)(b, c) \Phi(f)(x, a)=\Phi(g)(a, b) \\
& \times \sum_{i, j \geq 0}^{p} f\left(a_{i}, a_{j}\right) x^{i} c^{j}-\Phi(g)(a, c) \sum_{i, j \geq 0}^{p} f\left(a_{i}, a_{j}\right) x^{i} b^{j}+\Phi(g)(b, c) \sum_{i, j \geq 0}^{p} f\left(a_{i}, a_{j}\right) x^{i} a^{j} \\
& =\sum_{i, j, k, m \geq 0}^{p}\left\{g\left(a_{m}, a_{i}\right) f\left(a_{k}, a_{j}\right)-g\left(a_{m}, a_{j}\right) f\left(a_{k}, a_{i}\right)+g\left(a_{i}, a_{j}\right) f\left(a_{k}, a_{m}\right)\right\} a^{m} b^{i} c^{j} x^{k} .
\end{aligned}
$$

Since $f(x, y) \in \operatorname{Ker} \mathcal{L}_{3}^{(g)}$, it follows that

$$
g(a, b) f(x, c)-g(a, c) f(x, b)+g(b, c) f(x, a)=0 .
$$

Setting $a=a_{m}, b=a_{i}, c=a_{j}, x=a_{k}$ simultaneously in this identity yields that

$$
g\left(a_{m}, a_{i}\right) f\left(a_{k}, a_{j}\right)-g\left(a_{m}, a_{j}\right) f\left(a_{k}, a_{i}\right)+g\left(a_{i}, a_{j}\right) f\left(a_{k}, a_{m}\right)=0,
$$

which leads to the desired result.

Precisely the same argument applied to the kernel $\Lambda_{3}[x, y]$ of Lagrange operator $\mathcal{L}_{3}$ yields the following result.

Theorem 4.3. Let $\Lambda_{3}[x, y] \subseteq K e r \mathcal{L}_{3}$ and let $\Phi$ be a polynomial transformation, defined as above. Then $\Phi\left(\Lambda_{3}[x, y]\right) \subseteq \Lambda_{3}[x, y]$, where $\Phi\left(\Lambda_{3}[x, y]\right)$ is the image of $\Lambda_{3}[x, y]$ under $\Phi$.

\section{ACKNOWLEDGEMENTS}

Thanks are due to Professor L. Zhu and L.C. Hsu for helpful comments on earlier versions of this manuscript. Thanks are also due to the anonymous referee for his very detailed comments, in particular the idea of how to derive the Krattenthaler formula from Warnaar's matrix inversion.

\section{REFERENCES}

1. G.E.Andrews, q-series: their development and application in analysis,number theory, combinatorics, physics and computer algebra, NSF CBMS Regional Conf. Series, Vol 66, 1986. MR0858826 (88b:11063)

2. D.M.Bressoud, A matrix inverse, Proc. Amer. Math. Soc. 88 (1983), 446-448. MR0699411 (84g:33003)

3. L.Carlitz, Some inverse relations, Duke Math. J. 40 (1973), 893-901. MR0337651 (49:2420)

4. W.C.Chu, L.C.Hsu, Some new applications of Gould-Hsu inversion, J. Combin. Inform. System Sci. 14 (1989), 1-4. MR.1068649 (92c:05018)

5. I.B.Frenkel, V.G.Turaev, Elliptic solutions of the Yang-Baxter equation and modular hypergeometric functions, in V. I. Arnold et al. (eds.), The Arnold-Gelfand Mathematical Seminars, 171-204, Birkhauser, Boston, 1997. MF,1429892 (98k:33034) 
6. I.Gessel, D.Stanton, Applications of q-Lagrange inversion to basic hypergeometric series, Trans. Amer. Math. Soc. 277 (1983), 173-201. MR0690047 (84f:33009)

7. H.W.Gould, L.C.Hsu, Some new inverse series relation, Duke Math. J. 40 (1973), 885-891. MR0337652 (49:2421)

8. C.Krattenthaler, Operator methods and Lagrange inversion, a unified approach to Lagrange formulas, Trans. Amer. Math. Soc. 305 (1988), 431-465. MR0924765 (89d:05017)

9. C.Krattenthaler, A new matrix inverse, Proc. Amer. Math. Soc. 124 (1996), 47-59. MR1291781 (96d:15004)

10. C.Krattenthaler, M.Schlosser, A new multidimensional matrix inversions with applications to multiple q-series, Discrete Math. 204 (1999), 249-279. MR,1691873 (2000j:33011)

11. Xinrong Ma, A short proof of Krattenthaler formulas, Acta Math. Sin.(Engl. Ser.) 18 (2002), no. 2, 289-292. MR 1910964 (2003b:05009)

12. S.C.Milne, G.Bhatnagar, A characterization of inverse relations, Discrete Math. 193 (1998), 235-245. MR1661371 (2000a:05019)

13. J. Riordan, Combinatorial identities, John Wiley \& Sons, Inc., New York-London-Sydney 1968. MR0231725 (38:53)

14. H.Rosengren, M.Schlosser, On Warnnar's elliptic matrix inversion and Karlsson-Minton-type elliptic hypergeometric series, J. Comput. Appl. Math. 178 (2005), 377-391.

15. M.Schlosser, Multidimensional matrix inversions and $A_{r}$ and $D_{r}$ basic hypergemetric series, Ramanujan J. 1 (1997), 243-274. MR1606918 (99b:33036)

16. M.Schlosser, Some new applications of matrix inversions in $A_{r}$, Ramanujan J. 3 (1999), 405-461. MR1738906 (2001c:33032)

17. V.P.Spiridonov, Theta hypergeometric series, in V. A. Malyshev and A. M. Vershik (eds.), Asymptotic Combinatorics with Applications to Mathematical Physics, 307-327, Kluwer Acad. Publ., Dordrecht, 2002. MR2000728 (2004j:33012)

18. S.O.Warnaar, Summmation and transformation formulas for elliptic hypergeometric series, Constr. Approx. 18 (2002), 479-502. MR1920282 (2003h:33018)

19. E.T.Whittaker, G.N.Watson, A course of modern analysis, Reprint of the fourth (1927) edition, Cambridge University Press, Cambridge, 1996. MF1424469 (97k:01072)

Department of Mathematics, SuZhou University, SuZhou 215006, People's Republic OF CHINA

E-mail address: xrma@public1.sz.js.cn 